\title{
Charging electric vehicles in the smart grid
}

\author{
Chris Develder, Matthias Strobbe, Klaas De Craemer and Geert Deconinck
}

\begin{abstract}
High level challenges that motivate the evolution towards smart grids include (i) the anticipated electrification of transportation, including electrical vehicles (EVs), and (ii) the increasing penetration of distributed renewable energy sources (DRES). This chapter will discuss how the extra grid load stemming from the EVs can be handled, including the context of reduced control over power generation in light of DRES adoption (especially solar and wind power). After a basic introduction to common EV charging technology, we give two illustrative examples of controlling EV charging: avoiding peaks, and balancing against renewable generation. We then qualitatively present possible demand response (DR) strategies to realize such control. Finally, we highlight the need for, and underlying principles of, (smart grid) simulation tools, e.g., to study the effectiveness of such DR mechanisms.
\end{abstract}

\section{Introduction}

The transition of today's electricity grid towards a smart grid is driven by the need to make electricity delivery more reliable, economical and sustainable. The challenges ahead stem from (i) increased electrification, as well as (ii) higher penetration of

\footnotetext{
Chris Develder

Ghent University - iMinds, Ghent, Belgium, e-mail: chris.develder@intec.ugent.be

Matthias Strobbe

Ghent University - iMinds, Ghent, Belgium e-mail: matthias.strobbe@intec.ugent.be

Klaas De Craemer

Vito - EnergyVille, Antwerp, Belgium, e-mail: klaas.decraemer@vito.be

Geert Deconinck

KULeuven - EnergyVille, Leuven, Belgium, e-mail: geert.deconinck@kuleuven.be
} 
renewable energy sources (RES). Examples of (i) include the electrification of the electrical vehicle fleet, or heat pumps.

One challenge of (ii), i.e., RES such as wind turbines and solar panels, is their location: they are very much distributed (DRES), and typically connected in the distribution grid (low or medium voltage). This is in sharp contrast to more classical generation such as hydro plants, or the less environmentally friendly coal, gas or nuclear plants. These distribution grids (especially the low voltage portions) however have not been designed for the resulting power flows, which are potentially bidirectional. The power injection at the points of connection of the DRES may lead to voltage variations and even violation of the admissible voltage boundaries (e.g., beyond the maximal voltage limit). Further, when a feeder is disconnected from the grid, the generation within the islanded feeder may still be there, thus leading to unsafe scenarios both for equipment and people.

Another challenge obviously is the intermittent, uncontrollabe generation from DRES: we have no influence on how hard the wind blows, or the sun shines, and thus we note a paradigm shift from steering generation to controlling the load. This calls for scalable algorithms to achieve demand response (DR), e.g., by assessing the possible flexibility that can be exploited by shifting (a portion of the total) consumption in time. Another option would be to decouple production and consumption of power in time, through storage. Still, the latter for now is not cost effective yet for a massive rollout, e.g., in the distribution grid at each household or even aggregated per feeder.

Thus, some major challenges for the transition to smart grids are (i) the development of scalable control algorithms (potentially ranging from fully distributed to more centralized), (ii) the introduction of and reliance on information and communication technology (ICT) infrastructure and its interworking with the power grid, and (iii) the creation and implementation of new business and market models. It is clear that these challenges are not only technical, but also may require adaptation of regulatory frameworks.

The focus of this chapter will be on electrical vehicles (EVs), in terms of how to cope with the additional load on the power grid they entail, as well as how their charging can be optimized to maximally exploit RES power. Section 2 will first provide an overview of EV technology, the charging process, and the communication options for exchanging the charging control information. Next, we present two illustrative case studies: Section 3 considers controlling EV charging to avoid peaks in a residential distribution feeder, while Section 4 studies balancing the wind power generation with a fleet of EVs. In Section 5, we then give a more general overview of possible strategies for demand response algorithms. Lastly, Section 6 indicates the main ideas of smart grid simulation tools to study such cases. Section 7 then summarizes the conclusions. 


\section{Electrical vehicle charging}

Electric vehicles (EVs) can be implemented as hybrid electric vehicles (HEVs) or Battery Electric Vehicles (BEVs). Both contain a battery to power an electric motor, but a Hybrid Electric Vehicle also contains an internal combustion engine (ICE) that can recharge the battery or operate as a range extender. BEVs and Plugin HEVs (PHEVs) are charged through the electric power system, which can lead to increased congestion of the grid. Especially the impact on the low voltage grid can be significant, if the peak of arriving EVs that plug in to charge at home corresponds to the residential load peak (Clement-Nyns et al (2010); Gomez and Morcos (2003); see also Section 3).

At the same time, while the charging of EVs requires a large amount of energy, vehicles tend to be stationary during long periods, for example during the night or working hours. This creates the opportunity to spread the charging of the batteries in time and thereby limit their impact on the distribution grid. Coordinated charging of electric cars in a smart grid is an excellent application of large-scale demand response of (domestic) appliances, and is the focus of a lot of current research. Before moving to some illustrative case studies, this section summarizes the typical charging process.

\subsection{Battery charging and state of charge}

The charging process of an EV is primarily determined by the properties of its battery pack (NiMH, Li-ion variants, etc.). The battery capacity of contemporary PHEVs varies from 4 to $20 \mathrm{kWh}$, while that of EV batteries rather lies between 15 and $35 \mathrm{kWh}$.

Because of concerns of accelerated degradation, battery cells are not used between 0 and $100 \%$ of their potential energy storage capacity: state-of-charge (SOC) levels close to empty and full put the highest strain on the cells. During charging, and at high SOC states, the amount of current that can be "sinked" into the pack is limited by the maximum voltage that can safely be applied over the cells (Panasonic, 2007). If a cell is overvolted, chemical reactions occur that can permanently damage the cell. As a consequence, a high SOC also hinders brake energy recuperation. Similarly, too low a cell voltage leads to a progressive breakdown of its electrodes.

Because of the aforementioned risks, the charging process is controlled and guarded by a Battery Management System (BMS). During charging, a BMS will vary power depending on the SOC (Seljeseth et al, 2013; Kapoor, 2012). Typically, there is a constant current (CC) and a constant voltage (CV) phase, pictured conceptually in Fig. 1(a). During the CV mode, charging power decreases quickly and the amount of energy that is stored into the cell during this phase is relatively small. 


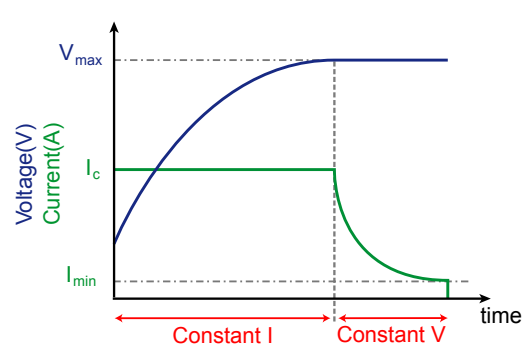

(a) Charging process

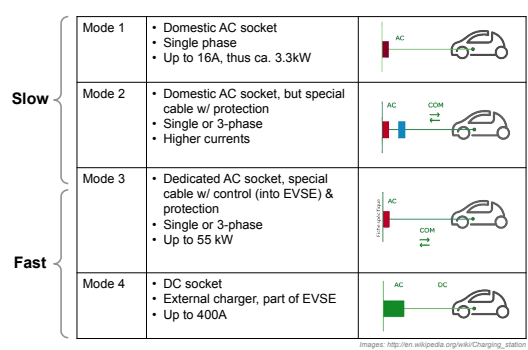

(b) Charging modes

Fig. 1 Electrical vehicle charging.

\subsection{IEC charging modes}

IEC 62196 (IEC Technical Committee 23, 2011) defines plugs, socket outlets, vehicle connectors and vehicle inlets. The charging modes, as summarized in Fig. 1(b), are referenced in IEC 61851-1:

- Mode 1 provides basic charging capabilities for domestic use, such that standard electrical plugs and outlets can be used (e.g., the CEE "Schuko" plug in Europe). The current in this mode is at most $16 \mathrm{~A}$, meaning that for a single phase connection, charge power is limited to $\sim 3.3 \mathrm{~kW}$.

- Mode 2 allows for higher charge currents, but imposes additional safety measures on the vehicle port and charging cable. A control pilot (CP) pin in the charging cable or in-line control box and the vehicle's charge connector is used to indicate the maximum charge current supported by the cable. Detection for proper earthing is also required.

- Mode 3 defines (fast) charging using an AC connection up to $55 \mathrm{~kW}$ and requires the use of dedicated EVSE (Electric Vehicle Supply Equipment), such as a wallbox. The requirement of a proximity pin (PP) with a shorter length ensures that a sudden disconnection or interruption is detected and the cable becomes unpowered.

- Mode 4 describes fast charging using DC, with an external charger. Work on standardization of DC charging is underway in IEC 62196-3.

\subsection{Communication}

All plugs and sockets, with the exception of the residential CEE "Schuko" plug (Mode 1), are designed to allow communication between the EV and the grid equipment (i.e., electrical vehicle supply equipment, EVSE), transferring charge power settings and schedules, as indicated in the right part of Fig. 1(b). An overview using early standards can be found in Ruthe et al (2011). 
- EVSE to EV: IEC 61851-1 defines a low level signaling protocol over the control pin (CP). Signaling from the EVSE to the vehicle is performed using a $1 \mathrm{kHz}$ PWM signal, from which the duty cycle is varied to indicate the current capabilities of the charging station.

- EV to EVSE: The EV can also send state information to the EVSE by switching load impedances between CP-PE (Control pin, Protected Earth; see Lewandowski et al (2011)). The EV's charger can indicate whether it is ready for charging or that ventilation is required during the charging process.

Also higher layer protocols exist (such as being specified in IEC 15118), which allow applications related to identification, payment, load leveling and value-added services. The Open Charge Point Protocol (OCPP) and IEEE P2030.1 allow communication between the EVSE or charge pole and a back office (e.g., an aggregator that coordinates the charging of a whole fleet).

\subsection{Alternative charging solutions}

Besides conductive charging (via a cable), alternative solutions exist, such as battery swapping or inductive charging. These solutions are not widely spread in the market yet, but provide similar opportunities for demand response as in case of conductive charging.

\section{Sample case study 1: Load flattening}

Now that we have outlined the main EV charging approaches, we venture into a first analysis of the impact of EV charging on the grid, in terms of total power consumption in a residential low voltage grid. In particular, we address the following high level questions:

1. What is the impact of uncontrolled EV charging in a residential environment?

2. What is the minimal impact on peak load that we could theoretically achieve?

3. How can we minimize the impact of EV charging in practice?

We explore these questions in a case study, detailed by Mets et al (2012a), in a three-phase distribution feeder comprising 63 households, which each have a single phase connection. The base load of the houses-that is, the total power consumption minus the EV-is set to that of a typical winter day in Flanders (which is the time of year with maximal electricity consumption), as taken from actual measurements. As for the EVs, we consider three scenarios: light, medium and heavy, that represent increasing EV adoption.

To answer the first question, we define a "business-as-usual" scenario (BAU), where drivers plug in their EVs when they arrive at home, and then the charging immediately starts. This scenario clearly needs no additional infrastructure beyond 


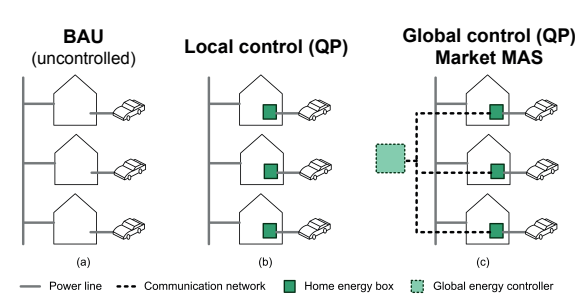

(a) Charging scenarios

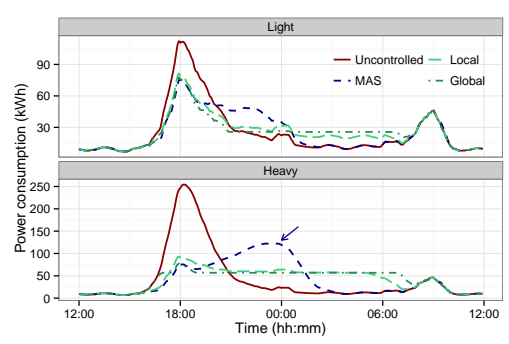

(b) Results

Fig. 2 Sample case study 1: Load flattening.

the charging equipment: no communication or control is needed. Yet, without any such control, power consumptiom from EV charging after arrival at home adds to the already existing peak in the base load profile, related to other human activities (cooking, appliance usage, hot water, etc.). In the particular case assumed by Mets et al (2012a), the increase in peak load due to EV charging amounts to between $1.5 \times$ and over $3 \times$ the original base load peak, i.e., a increase in the same order of magnitude as the original peak. We note that also in terms of total yearly power consumption, the energy used for charging a full EV is in the same range of a typical household's energy from the non-EV base load. Clearly, to avoid the need to reinforce the grid with extra generation (to meet the peak load), spreading the EV charging in time is needed - as to minimize coincidence with the already existing peak load. We note that apart from peak load reduction, spreading the EV charging in time also may help to avoid, e.g., over voltages. Indeed, when excessive power is being drawn at certain connection points, the voltage may drop to levels outside (i.e., below) the tolerable bounds around the nominal voltage. (We will not dwell on the latter point here; see Mets et al (2012a).)

Now, we investigate the theoretical bounds in terms of minimizing peak load by shifting/spreading the EV charging in time: since most cars will stay connected all night before leaving in the morning, we do not need to start charging immediately upon arrival. We adopt quadratic programming $(Q P)$ to formulate an optimization problem that tries to shift around (EV charging) power consumption as to obtain a flat power consumption over time. The approach is theoretical, in the sense that it assumes all events (cars arriving and departing, the base load of households) are fully and correctly known. We consider two fundamentally different variants: (i) local control only uses knowledge of the household itself (in terms of both base load and EV), while (ii) global control relies on knowing also the state of other households (load and EVs).

Note that these fundamentally different assumptions also imply distinct infrastructure need to enable them in practice, as illustrated in Fig. 2(a). Indeed, the local approach only needs local technology to gather the data (e.g., arrival of the EV, and when it will leave again): no communication with other entities is strictly necessary. The global control approach needs at least a channel to reach other subscribers, ei- 
ther directly or through a centralized control entity - the latter depends on whether we realize the global control either as a fully distributed coordination strategy, or rather as a centralized one (see Section 5 for a discussion of the types of DR algorithms).

To move away from such bounds on what is achievable when we would be allknowing (i.e., have perfect knowledge of past/present/(short) future EV and base load behavior), and rather get an idea of what is realistically achievable, we consider a market-based coordination mechanism, based on multi-agent systems (MAS). In particular we consider a single-shot, multi-unit auction market mechanism (see Mets et al, 2012b). This means that the control signal that will steer power consumption, is a price signal. (That control price signal may be directly tied to actual monetary prices to be paid by consumers, but it could equally be purely virtual, i.e., just a means of control that could be completely decoupled from the billing of energy usage.) The general principle of the market-based MAS is that participating consumer entities, i.e., the EV chargers in our case, have a price response function, also known as a bidding function. Such bidding function determines what the power consumed will be for a given price. This function may change over time: e.g., for $\mathrm{EVs}$, as the deadline for completing the charging approaches, the willingness to use power will increase and even for high(er) price signals charging will happen. The advantage of such a market based system is that the control signal is very simple, and the approach scales very well. Still, the design of the bidding curves is not a trivial exercise, and in our EV case it still assumes that the state-of-charge of the battery is perfectly known.

An illustrative comparison between the various cases is given in Fig. 2(b). First, we note that all control approaches, both the theoretical (Local QP, Global QP) and more realistic ones (MAS) succeed in moving away the peak from that of the base load: the peak around 18:00 is entirely caused by the base load. Second, comparing Local vs Global, we note that in theory, even with just local knowledge (i.e., no communication beyond the household) the charging process can be controlled to successfully flatten the resulting total load profile of the entire feeder. Yet, we note that in our experiment the correlation between various households was quite high - whether in reality that will mostly be the case (or to the same extent as in our limited experiment) is debatable. Third, we note that depending on the EV penetration, the MAS system with the chosen linear bidding curves manages quite well in approaching the theoretical Local/Global QP boundary. Still, in case of the "heavy" scenario, we note the appearance of a new peak around midnight (indicated with the arrow): this illustrates that the design of the bidding functions can/should be tuned to the particular (amount of) interacting entities.

\section{Sample case study 2: Balancing renewable generation}

In the previous section we focused on dealing with the additional load stemming from EV charging, as to not aggravate the consumption peaks. Realizing that EV 
charging can be spread in time, now we investigate how well we can exploit that time shifting of charging to try and meet the intermittent production from renewable energy sources (over which we clearly have no full control). Apart from ecological motivations (i.e., to maximally exploit RES and avoid less environmentally friendly sources), there are also technical incentives to try and balance the RES production. For example, if RES generation is dispersed along a distribution feeder, the injection may raise the voltage level at the point of connection beyond the admissible limit, and thus create over-voltage problems.

In the illustrative case study (taken from Mets et al, 2012a) that we will now summarize, we consider balancing power supplied by a small scale wind turbine. In particular, we propose a distributed algorithm to balance renewable energy from wind turbines with the charging demand of electric vehicles, thereby increasing renewable energy consumption, and reducing emissions of greenhouse gasses. We approach this problem from the viewpoint of a balance responsible party (BRP), also known as access responsible party (ARP), that is responsible to ensure that energy supply matches energy consumption during a given time period: if the balance is not maintained, the BRP is required to pay imbalance costs. Therefore, the objective of the BRP is to minimize the imbalance costs. Nevertheless, the wishes and preferences of subscribers have to be respected, and are therefore accounted for in our approach, while maintaining privacy by not sharing their detailed consumption information directly (e.g., they do not share arrival and departure times, vehicle properties, nor the willingness to participate in balancing demand and supply).

We consider the following participants in our distributed coordination algorithm: (i) subscribers represent the EV charging spots, (ii) the BRP knows the wind generation (i.e., its predicted output), and thus the target consumption profile required to achieve balancing, (iii) the coordinator is the core component that will communicate with all previous parties and align them. Note that our case study here assumes only a single coordinator, but to further scale up, we can deploy also multiple coordinators, each managing their own set of subscribers. Similarly, one can introduce intermediate aggregator components, grouping together a set of subscribers and thus form an intermediate level (between subscribers and coordinator) in a hierarchical constellation. We will not discuss such more advanced setups here.

The algorithm we propose is an example of a receding horizon control algorithm. We assume a time slotted approach, say with 15 min timeslots. Every timeslot $t$, a control algorithm is executed considering the next $T$ timeslots. As in our load flattening case, the control signal again is a virtual price signal. The steps are summarized as:

1. Initialization:

a. The BRP updates the wind power generation forecast $\mathbf{w}=\left[w_{t}, \ldots w_{t+T}\right]$ for the next $T$ time slots.

b. The coordinator initializes a (virtual) price vector $\mathbf{p}=\left[p_{t}, \ldots p_{t+T}\right]$ used to steer demand and supply.

2. The coordinator sends the price vector $\mathbf{p}$ to the subscribers and the BRP. 
3. Each subscriber calculates an power consumption schedule based on $\mathbf{p}$ and its own requirements and preferences, and sends that schedule to the coordinator.

4. The BRP determines an energy production schedule based on the wind power generation forecast and $\mathbf{p}$, and sends it to the coordinator.

5. The coordinator collects the consumption schedules from the subscribers and production schedules from the BRP, and compares them:

a. If the discrepancy between supply and demand is below a predefined acceptance level, or the maximum number of iterations is reached, the algorithm terminates: subscribers and BRP are notified that the schedules are final.

b. Else, the coordinator updates the price vector $\mathbf{p}$ and iterates from step 2 .

The mathematical materialization of this algorithm relies on dual decomposition to split the overall optimization problem in sub-problems that can be solved independently (and thus in parallel, distributed over multiple participants). We refer to Mets et al (2012a) for details.

We applied that algorithm to a case study comprising 100 electric vehicles (with arrivals, departures and state-of-charge of the battery upon arrival that are derived from a statistical model of real-life vehicle usage data), and a small wind turbine with peak power output of $30 \mathrm{~kW}$ (whose the total power production over time sligthly exceeds the required charging power aggregated over time). Illustrative results are shown in Fig. 3.

We compare the above distributed algorithm with two baselines (see Fig. 3): (i) a business as usual (BAU) scenario, where EVs are charged at full power as soon as they arrive, and (ii) an "ideal world" benchmark that fully minimizes imbal-

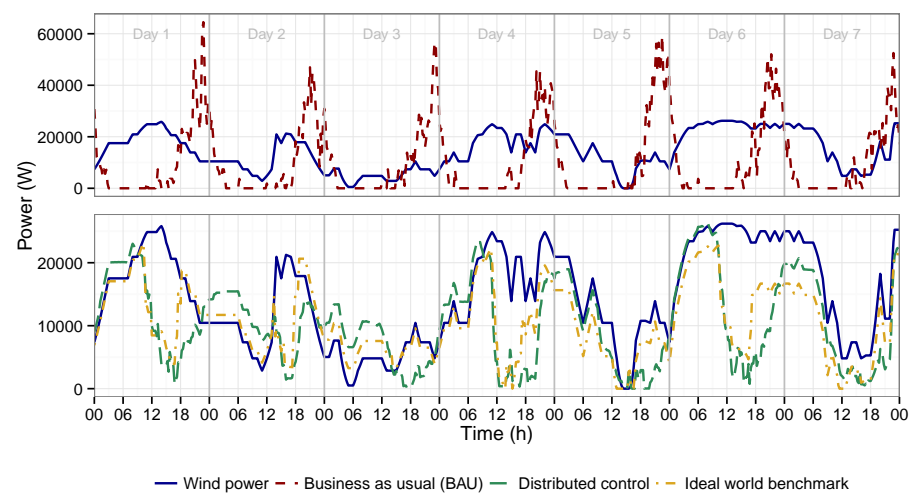

Fig. 3 Sample case study 2: Balancing renewable generation. Note that the Wind power curves in both plots are the same: controlled charging scenarios are shown with a different Y-axis scale to better distinguish them. 
ance $^{1}$, which is all-knowing. The latter implies that the benchmark approach has full and exact knowledge of both wind power production and EV arrivals, departures and state-of-charge. Comparing our algorithm's results to the BAU scenario, we note that it clearly succeeds in avoiding the peaks in consumption, while reasonably matching the wind power generation profile. Whether we could in theory do much better can be seen by comparing to the benchmark: we note that the match is pretty good. The result of applying our algorithm is that the fraction of power supplied from the renewable source increases from $40 \%$ in the BAU case to $68 \%$ (while the theoretical benchmark reaches $73 \%$ ). This means that we can reduce the $\mathrm{CO}_{2}$ emissions of the BAU case with about $45 \%$. Thus, we note that distributed coordination is a viable approach to tackle the challenge of shaping the consumption to renewable production. The next section will give a broader overview of possible strategies for such so-called demand response (DR) implementations.

\section{Demand response strategies}

Current research regarding the optimization and coordination of clusters of DR participants can roughly be divided according to the way the optimization is performed: distributed, centralized and aggregate \& dispatch algorithms, as illustrated in Fig. 4(a).

Distributed algorithms perform a significant part of the optimization process of allocating energy over the cluster at the participating devices themselves. This way, the computational complexity of finding a suitable solution is spread out over the demand response cluster, typically using an iterative process where information is communicated between the participants. However, the distributed aspect does not exclude the existence of an entity responsible for initiating or coordinating the convergence over the iterations. Additionally, while possible, the implementation of a distributed algorithm is not necessarily in a peer-to-peer-style fashion, as would be suggested by Fig. 4(a).

Centralized algorithms are entirely the opposite. A central actor collects information that is sent to it from the DR devices. This information can consist of individual constraints and deadlines or comfort settings. Using the collected knowledge, and possibly including its own additional information such as predictions or stochastic functions, the central coordinator can perform a single optimization that returns an optimal schedule satisfying all the constraints at once. Inherently, this makes centralized algorithms the least scalable, as the optimization process quickly becomes intractable with an increasing number of participating devices. Furthermore, the communication towards and from a single point poses a potential bottleneck. Several solutions are proposed that help to overcome the tractability issue.

In between distributed and centralized mechanism are the aggregate $\&$ dispatch algorithms. They decouple the optimization of the objective and the dispatch of its

\footnotetext{
${ }^{1}$ This imbalance is formulated as sum of squared differences between generation and consumption,
} summed over all timeslots. 
outcome, thus alternatively the term dispatching mechanism is equally fitting. An aggregate \& dispatch mechanism allows information (such as constraints) from and to the central entity to be aggregated, reducing the complexity of the optimization and improving scalability, but carrying certain compromises or constraints regarding the optimality of the results.

Centralized algorithms provide a way to incorporate a large amount of diverse information and constraints in the DR scheduling problem, which can then be solved by well-established mathematical techniques. This guarantees that the outcome is optimal with respect to the problem's constraints. However, due the complexity involved, the time needed for solving quickly spirals out of control when scaling to large clusters of devices or when more advanced scenarios are taken into account. This is referred to as the curse of dimensionality, and can be partly addressed by the use of approximation and search techniques.

As an alternative, the DR scheduling problem can be broken down so that it can be distributed over multiple participants in the DR cluster (e.g., De Craemer et al, 2014). A method such as dual decomposition works by iteratively exchanging demand information and coordination signals between a central entity and the cluster's autonomous devices until convergence is reached. Alternatively, the use of game theory can provide proofs regarding the fairness of the scheduling process. The downside of the distributed algorithms is related to the need to exchange additional messages between the devices (since either multiple iterations are required or they communicate directly with each other) and the additional complexity involved due to the requirements for the communication system.

The division of the algorithms into centralized and distributed is also loosely tied to the control architecture in which they would be implemented. A centralized algorithm will have a single entity where all data for the optimization is collected and coordination signals to the individual DR devices is sent out from. In the distributed case, devices are more autonomous and may even communicate in peer-to-peer fashion.

An alternative to the centralized and distributed algorithms is provided by aggregate $\&$ dispatch algorithms. These methods use an aggregated model to represent or approximate the collective state of the DR cluster. The model is updated with state information from the individual devices. Scheduling then takes place using

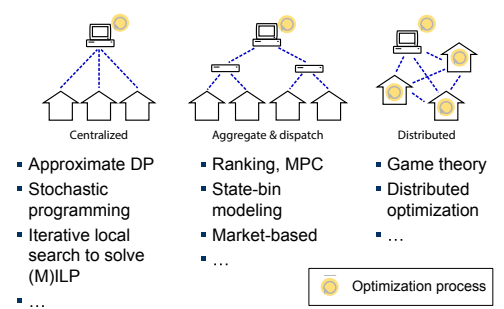

(a) Taxonomy

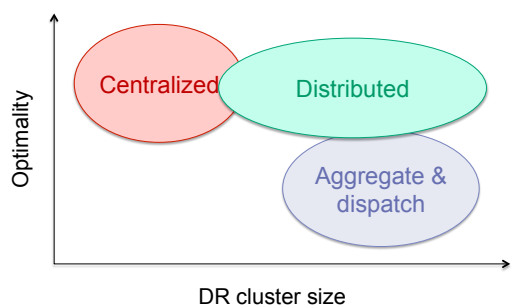

(b) Performance tradeoffs

Fig. 4 Demand side management strategies. 
the aggregated model and the result is dispatched to the DR devices through, e.g., heuristic methods. Because the aggregated model fails to capture some of the details of the individual devices' state and heuristics are not perfect, aggregate \& dispatch methods do not achieve the most optimal schedule. However, they obtain results that are close to the centralized or distributed algorithms, but at much lower complexity, and scale well to DR clusters containing large amounts of devices. They constitute a good trade-off, as they can achieve most of the benefits of DR at a large scale, but at low complexity and consequently cost.

In light of the above description, Fig. 4(b) positions the three classes of DR algorithms in terms of scalability and optimality.

\section{Simulation tools}

Simulation tools offer a cost effective and safe approach to assess the performance of demand response control strategies and other smart grid use cases (such as the ones presented in Sections 3 and 4). Different solutions can be evaluated under varying conditions before actually deploying them in the field, as to study the complex interactions between communication networks and power systems, and the monitoring and control elements on top of them.

Within smart grid research three groups of applicable tools can be distinguished: (i) power system simulators, (ii) communication network simulators, and (iii) smart grid simulators, where the last category combines the simulation of both the power grid and communication infrastructure.

Power simulation tools can be largely divided into two classes: they are either targeted at steady state analysis (typically power flow studies), or at transient dynamics simulations (typically upon disturbances or sudden system changes). They typically adopt a continuous time model, studying the system state at fixed points, regularly spaced over time.

Communication network simulators on the other hand typically adopt a discrete event simulation approach, where time intervals between successive events (i.e., system changes) can greatly vary. Depending on the focus of the study at hand, communication network can be modeled as (i) a black box model, a simplified and abstract model of the simulated communication network with only a few parameters (e.g., delay, packet loss, bandwidth) or (ii) as a detailed communication network model with an accurate topology containing hosts, switches, routers, etc., and models for the full networking stack (from application to physical layer).

Two high level approaches to combine both power and communication simulators are illustrated in Fig. 5. In case of co-simulation distinct simulators are used for the communication network and power grid, each having their own interface for data input, configuration, result output, control, etc. This approach requires careful synchronization of data and interactions between both simulators, especially with respect to time management, because each simulator manages its simulation time individually. This can be realized using predefined synchronization points where 


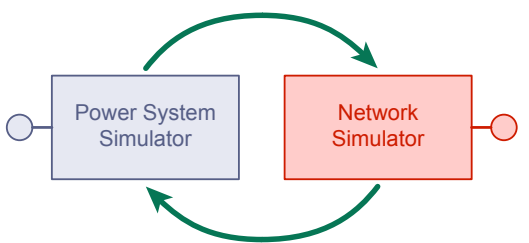

(a) Co-simulation

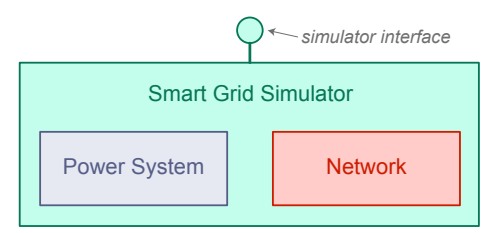

(b) Integrated simulation

Fig. 5 Conceptual approaches to combining power and communication network simulation.

both simulators pause and information is exchanged. An advantage of this approach is that existing simulation models, algorithms, etc. can be reused. In this approach typically one simulator is selected as a master for the synchronization logic. This master usually also contains the control logic for the simulated use case.

In integrated or comprehensive simulation, both the power system and communication network are simulated in one environment. A single interface is provided and the management of time, data, and power/communication system interactions can be shared among the simulator constituents. Hence, no performance penalty due to synchronization is expected. The main challenge is the combination of both models in one environment and to provide a simulation interface that provides sufficient level of detail for the different aspects of the smart grid simulation model.

Other important requirements for smart grid simulators are the need for new models to characterize for example renewable energy sources, to correctly deal with their intermittent and stochastic behavior. For DR approaches, the correct modeling of the user behavior, and especially the flexibility of his load (e.g., charging deadlines for EVs) is crucial. Simulators should be open, user-friendly and flexible environments, that support user defined models, and easy reuse of already established and validated models.

Most smart grid simulation tools are purely software-based solutions. Yet, other approaches aim for more realism. Emulated components more closely mimic the real world in hardware, e.g., a communication network in a lab consisting of real hosts, routers and network cables. In real-time simulation the simulation clock is synchronized with the real-time clock, which might be necessary to assess the timeliness with which the model interacts with external components (e.g., for protection), e.g., for Hardware-in-the loop (HIL) simulation, which combines real hardware with simulated components.

Many of the currently available smart grid simulators focus on specific use cases, providing answers to specific research questions. Still, a few more generic simulation environments that support a wide range of use cases and are much more extensible. In this respect, federated simulators are a promising approach for co-simulation as they allow the easy addition of extra components (e.g., external data sources with weather or traffic information) and support distributed simulation. For a more detailed discussion of simulation for smart grid applications, we refer to Mets et al (2014). 


\section{Conclusions}

Current electrical vehicles (EVs) relay on a charging process to replenish the power of batteries. While alternatives have been proposed (e.g., battery swapping), the majority of EVs today rely on charging by wire, either using a standard household plug, or dedicated special-purpose EV plugs and charging infrastructure. The power consumption associated with a single EV on a yearly basis lies in the same range as that of a complete household. Besides the overall larger volume of energy required, uncontrolled charging (e.g., in a residential scenario, starting the charging as soon as the user with his EV arrives at home) would also add on to the typical consumption peak in the evening. This can be solved bscaly peak flattening through demand response (DR) approaches; similar algorithms can also be used for balancing. We advocate for the use of distributed algorithms, since these form a good tradeoff between optimality (e.g., in attaining the balancing objective) and scalability (over very large user populations). To bring these algorithms (from mostly theoretical studies) to the real world, careful validation is still required. Further development (e.g., scaling up) of simulation tools that combine both the power grid, the communication network and the actual smart grid applications is required before moving to proof-of-concept trials, but also or to complement them with larger scale experiments.

Acknowledgements The work presented in this chapter has been supported in part by the European Commission through the project P2P-Smartest: Peer to Peer Smart Energy Distribution Networks (H2020-LCE-2014-3, project 646469).

\section{References}

Clement-Nyns K, Haesen E, Driesen J (2010) The impact of charging plug-in hybrid electric vehicles on a residential distribution grid. IEEE Trans Power Sys 25(1):371-380, DOI 10.1109/TPWRS.2009.2036481

De Craemer K, Vandael S, Claessens B, Deconinck G (2014) An event-driven dual coordination mechanism for demand side management of PHEVs. IEEE Trans Smart Grid 5(2):751-760, DOI 10.1109/TSG.2013.2272197

Gomez J, Morcos M (2003) Impact of EV battery chargers on the power quality of distribution systems. IEEE Trans Power Deliv 18(3):975-981, DOI 10.1109/TPWRD.2003.813873

IEC Technical Committee 23 (2011) IEC 62196 ed2.0: Plugs, socket-outlets, vehicle connectors and vehicle inlets - Conductive charging of electric vehicles

Kapoor A (2012) Charging algorithms of lithium-ion batteries: An overview. In: 7th IEEE Conf. Industrial Electronics and Applications (ICIEA 2012), Singapore, pp 1567-1572, DOI 10.1109/ICIEA.2012.6360973 
Lewandowski C, Gröning S, Schmutzler J, Wietfeld C (2011) Performance evaluation of PLC over the IEC 61851 control pilot signal. In: 5th Workshop on Power Line Communications (WSPLC 2011), Arnhem, The Netherlands

Mets K, De Turck F, Develder C (2012a) Distributed smart charging of electric vehicles for balancing wind energy. In: Proc. 3rd IEEE Int. Conf. Smart Grid Communications (SmartGridComm 2012), Tainan City, Taiwan, pp 133-138, DOI 10.1109/SmartGridComm.2012.6485972

Mets K, D'hulst R, Develder C (2012b) Comparison of intelligent charging algorithms for electric vehicles to reduce peak load and demand variability in a distribution grid. J Commun Netw 14(6):672-681, DOI 10.1109/JCN.2012.00033

Mets K, Aparicio J, Develder C (2014) Combining power and communication network simulation for cost-effective smart grid analysis. IEEE Commun Surveys Tutorials 16(3):1771-1796, DOI 10.1109/SURV.2014.021414.00116

Panasonic (2007) Lithium Ion Batteries Technical Handbook. URL http://industrial.panasonic.com/www-data/pdf/ACI4000/ACI4000PE5.pdf

Ruthe S, Schmutzler J, Rehtanz C, Wietfeld C (2011) Study on V2G protocols against the background of demand side management. Int J Bus Inform Sys pp 33-44

Seljeseth H, Henning T, Solvang T (2013) Measurements of network impact from electric vehicles during slow and fast charging. In: Proc. 22nd Int. Conf. Electricity Distribution (CIRED 2013), Stockholm, Sweden, DOI 10.1049/cp.2013.1197 